\title{
ABELIAN VARIETIES OVER FIELDS OF FINITE CHARACTERISTIC
}

\author{
YURI G. ZARHIN
}

\begin{abstract}
The aim of this paper is to extend our previous results about Galois action on the torsion points of abelian varieties to the case of (finitely generated) fields of characteristic 2 .
\end{abstract}

\section{INTRODUCTION}

Let $K$ be a field, $\bar{K}$ its algebraic closure, $\bar{K}_{s} \subset \bar{K}$ the separable algebraic closure of $K, \operatorname{Gal}(K)=\operatorname{Gal}\left(\bar{K}_{s} / K\right)=\operatorname{Aut}(\bar{K} / K)$ the absolute Galois group of $K$.

Let $X$ be an abelian variety over $K$. Then we write $\operatorname{End}(X)$ for its ring of $K$-endomorphisms and $\operatorname{End}^{0}(X)$ for the finite-dimensional semisimple $\mathbf{Q}$-algebra $\operatorname{End}(X) \otimes \mathbf{Q}$. If $n$ is a positive integer that is not divisible by $\operatorname{char}(K)$ then we write $X_{n}$ for the kernel of multiplication by $n$ in $X(\bar{K})$; it is well known that $X_{n}$ is free $\mathbf{Z} / n \mathbf{Z}$-module of rank $2 \operatorname{dim}(X)$ [9, which is a Galois submodule of $X\left(\bar{K}_{s}\right)$. We write $\bar{\rho}_{n, X}$ for the corresponding (continuous) structure homomorphism

$$
\bar{\rho}_{n, X}: \operatorname{Gal}(K) \rightarrow \operatorname{Aut}_{\mathbf{Z} / n \mathbf{Z}}\left(X_{n}\right) \cong \mathrm{GL}(2 \operatorname{dim}(X), \mathbf{Z} / n \mathbf{Z}) .
$$

In particular, if $n=\ell$ is a prime different from $\operatorname{char}(K)$ then $X_{\ell}$ is a $2 \operatorname{dim}(X)$ dimensional $\mathbf{F}_{\ell \text {-vector space provided with }}$

$$
\bar{\rho}_{\ell, X}: \operatorname{Gal}(K) \rightarrow \operatorname{Aut}_{\mathbf{F}_{\ell}}\left(X_{\ell}\right) \cong \mathrm{GL}\left(2 \operatorname{dim}(X), \mathbf{F}_{\ell}\right) .
$$

We write

$$
\tilde{G}_{\ell}=\tilde{G}_{\ell, X, K}
$$

for the image (subgroup)

$$
\bar{\rho}_{\ell, X}(\operatorname{Gal}(K)) \subset \operatorname{Aut}_{\mathbf{F}_{\ell}}\left(X_{\ell}\right) .
$$

By definition, $\tilde{G}_{\ell, X, K}$ is a finite subgroup of

$$
\operatorname{Aut}_{\mathbf{F}_{\ell}}\left(X_{\ell}\right) \cong \mathrm{GL}\left(2 \operatorname{dim}(X), \mathbf{F}_{\ell}\right) .
$$

If $K\left(X_{\ell}\right)$ is the field of definition of all points of order $\ell$ on $X$ then it is a finite Galois extension of $K$ and the corresponding Galois group $\operatorname{Gal}\left(K\left(X_{\ell}\right) / K\right)$ is canonically isomorphic to $\tilde{G}_{\ell, X, K}$. If $K^{\prime} / K$ is a finite Galois extension of fields then $\operatorname{Gal}\left(K^{\prime}\right)$ is a normal open subgroup of finite index in $\operatorname{Gal}(K)$ while $X^{\prime}=X \times_{K} K^{\prime}$ is a $\operatorname{dim}(X)$-dimensional abelian variety over $K^{\prime}$ and the $\operatorname{Gal}\left(K^{\prime}\right)$-modules $X_{\ell}$ and $X_{\ell}^{\prime}$ are canonically isomorphic. Under this isomorphism, $\tilde{G}_{\ell, X^{\prime}, K^{\prime}}$ becomes isomorphic to a certain normal subgroup of $\tilde{G}_{\ell, X, K}$.

This work was partially supported by a grant from the Simons Foundation (\#246625 to Yuri Zarkhin). 
By functoriality, $\operatorname{End}(X)$ acts on $X_{n}$. This action gives rise to the embedding of free $\mathbf{Z} / n \mathbf{Z}$-modules

$$
\operatorname{End}(X) \otimes \mathbf{Z} / n \mathbf{Z} \hookrightarrow \operatorname{End}_{\mathbf{Z} / n \mathbf{Z}}\left(X_{n}\right) ;
$$

in addition, the image of $\operatorname{End}(X) \otimes \mathbf{Z} / n \mathbf{Z}$ lies in the centralizer $\operatorname{End}_{\operatorname{Gal}(K)}\left(X_{n}\right)$ of $\operatorname{Gal}(K)$ in $\operatorname{End}_{\mathbf{Z} / n \mathbf{Z}}\left(X_{n}\right)$. Further we will identify $\operatorname{End}(X) \otimes \mathbf{Z} / n \mathbf{Z}$ with its image in $\operatorname{End}_{\operatorname{Gal}(K)}\left(X_{n}\right)$ and write

$$
\operatorname{End}(X) \otimes \mathbf{Z} / n \mathbf{Z} \subset \operatorname{End}_{\operatorname{Gal}(K)}\left(X_{n}\right) .
$$

If $\ell$ is a prime that is different from $\operatorname{char}(K)$ then we write $T_{\ell}(X)$ for the $\mathbf{Z}_{\ell}$-Tate module of $X$ and $V_{\ell}(X)$ for the corresponding $\mathbf{Q}_{\ell \text {-vector space }}$

$$
V_{\ell}(X)=T_{\ell}(X) \otimes \mathbf{z}_{\ell} \mathbf{Q}_{\ell}
$$

provided with the natural continuous Galois action [13]

$$
\rho_{\ell, X}: \operatorname{Gal}(K) \rightarrow \operatorname{Aut}_{\ell}\left(T_{\ell}(X)\right) \subset \operatorname{Aut}_{\mathbf{Q}_{\ell}}\left(V_{\ell}(X)\right) .
$$

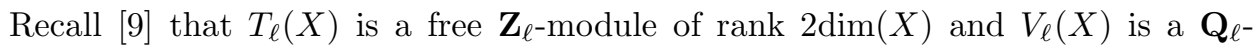
vector space of dimension $2 \operatorname{dim}(X)$. Notice that there are canonical isomorphisms of $\operatorname{Gal}(K)$-modules

$$
X_{\ell^{i}}=T_{\ell}(X) / \ell^{i} T_{\ell}(X)
$$

for all positive integers $i$. The natural embeddings

$$
\operatorname{End}(X) \otimes \mathbf{Z} / \ell^{i} \mathbf{Z} \hookrightarrow \operatorname{End}_{\mathbf{Z} / \ell^{i} \mathbf{Z}}\left(X_{\ell^{i}}\right)
$$

are compatible and give rise to the embeddings of $\mathbf{Z}_{\ell \text {-algebras }}$

$$
\operatorname{End}(X) \otimes \mathbf{Z}_{\ell} \hookrightarrow \operatorname{End}_{\mathbf{z}_{\ell}}\left(T_{\ell}(X)\right)
$$

and $\mathbf{Q}_{\ell}$-algebras

$$
\operatorname{End}(X) \otimes \mathbf{Q}_{\ell} \hookrightarrow \operatorname{End}_{\mathbf{Q}_{\ell}}\left(V_{\ell}(X)\right) .
$$

Again, the images of $\operatorname{End}(X) \otimes \mathbf{Z}_{\ell}$ in $\operatorname{End}_{\mathbf{z}_{\ell}}\left(T_{\ell}(X)\right)$ and of $\operatorname{End}(X) \otimes \mathbf{Q}_{\ell}$ in $\operatorname{End}_{\mathbf{Q}_{\ell}}\left(V_{\ell}(X)\right)$ lie in the centralizers $\operatorname{End}_{\mathrm{Gal}(K)}\left(T_{\ell}(X)\right)$ and $\operatorname{End}_{\mathrm{Gal}(K)}\left(V_{\ell}(X)\right)$ respectively. We will identify $\operatorname{End}(X) \otimes \mathbf{Z}_{\ell}$ and $\operatorname{End}(X) \otimes \mathbf{Q}_{\ell}$ with their respective images and write

$$
\begin{aligned}
\operatorname{End}(X) \otimes \mathbf{Z}_{\ell} \subset \operatorname{End}_{\mathrm{Gal}(K)}\left(T_{\ell}(X)\right) \subset \operatorname{End}_{\mathbf{z}_{\ell}}\left(T_{\ell}(X)\right), \\
\operatorname{End}(X) \otimes \mathbf{Q}_{\ell} \subset \operatorname{End}_{\mathrm{Gal}(K)}\left(V_{\ell}(X)\right) \subset \operatorname{End}_{\mathbf{Q}_{\ell}}\left(V_{\ell}(X)\right) .
\end{aligned}
$$

Similarly, if $Y$ is another abelian variety over $K$ then we write $\operatorname{Hom}(X, Y)$ for the (free commutative) group of all $K$-homomorphisms from $X$ to $Y$. Similarly, there are the natural embeddings

$$
\begin{aligned}
& \operatorname{Hom}(X, Y) \otimes \mathbf{Z} / n \mathbf{Z} \subset \operatorname{Hom}_{\operatorname{Gal}(K)}\left(X_{n}, Y_{n}\right) \subset \operatorname{Hom}_{\mathbf{Z} / n \mathbf{Z}}\left(X_{n}, Y_{n}\right), \\
& \operatorname{Hom}(X, Y) \otimes \mathbf{Z} / \ell^{i} \mathbf{Z} \subset \operatorname{Hom}_{\operatorname{Gal}(K)}\left(X_{\ell^{i}}, Y_{\ell^{i}}\right) \subset \operatorname{Hom}_{\mathbf{Z} / \ell^{i} \mathbf{Z}}\left(X_{\ell^{i}}, Y_{\ell^{i}}\right), \\
& \operatorname{Hom}(X, Y) \otimes \mathbf{Z}_{\ell} \subset \operatorname{Hom}_{\operatorname{Gal}(K)}\left(T_{\ell}(X), T_{\ell}(Y)\right) \subset \operatorname{Hom}_{\mathbf{Z}_{\ell}}\left(T_{\ell}(X), T_{\ell}(Y)\right), \\
& \operatorname{Hom}(X, Y) \otimes \mathbf{Q}_{\ell} \subset \operatorname{Hom}_{\mathrm{Gal}(K)}\left(V_{\ell}(X), V_{\ell}(Y)\right) \subset \operatorname{Hom}_{\mathbf{Q}_{\ell}}\left(V_{\ell}(X), V_{\ell}(Y)\right) . \\
& \text { Let } \\
& \rho_{\ell, X}: \operatorname{Gal}(K) \rightarrow \operatorname{Aut}_{\mathbf{z}_{\ell}}\left(T_{\ell}(X)\right) \subset \operatorname{Aut}_{\mathbf{Q}_{\ell}}\left(V_{\ell}(X)\right)
\end{aligned}
$$

be the corresponding $\ell$-adic representation of $\operatorname{Gal}(K)$. The image

$$
G_{\ell, X, K}=\rho_{\ell, X}(\operatorname{Gal}(K)) \subset \operatorname{Aut}_{\mathbf{z}_{\ell}}\left(T_{\ell}(X)\right) \subset \operatorname{Aut}_{\mathbf{Q}_{\ell}}\left(V_{\ell}(X)\right)
$$

is a compact $\ell$-adic Lie (sub)group [12, 13]. 
Let $d$ be a positive integer. We write $\operatorname{Isog}(X, K, d)$ for the set of $K$-isomorphism classes of abelian varieties $Y$ over $K$ that enjoy the following properties:

(i) $Y$ admits a $K$-polarization of degree $d$;

(ii) There exists a $K$-isogeny $Y \rightarrow X$ whose degree is prime to $\operatorname{char}(K)$.

For example, if $d=1$ then $\operatorname{Isog}(X, K, 1)$ consists of ( $K$-isomorphism classes of) all principally polarized abelian varieties $Y$ over $K$ that admit a $K$-isogeny whose degree is prime to $\operatorname{char}(K)$.

We write $\operatorname{Isog}(X, K)$ for the set of $K$-isomorphism classes of abelian varieties $Y$ over $K$ such that there exists a $K$-isogeny $Y \rightarrow X$ whose degree is prime to char $(K)$. Clearly, $\operatorname{Isog}(X, K)$ coincides with the union of all $\operatorname{Isog}(X, K, d)$ 's.

The following statement was proven under an additional assumption that $p$ does not divide $d$ by the author when $p>2[18$ and by S. Mori when $p=2[\underline{8}$, Ch. XII, Cor. 2.4 on p. 244]. (This is a strenghening of Tate' finiteness conjecture for isogenies of abelian varieties [16, 25].)

Theorem 1.1 (Corollary 2.4 on p. 244 of [8]). Assume that $p:=\operatorname{char}(K)>0$ and $K$ is finitely generated over the finite prime field $\mathbf{F}_{p}$. Let $d$ be a positive integer and $X$ be an abelian variety over $K$.

Then the set $\operatorname{Isog}(X, K, d)$ is finite.

The finiteness of $\operatorname{Isog}(X, K, d)$ combined with results of 17] implies the Tate conjecture on homomorphisms of abelian varieties and the semisimplicity of Tate modules over $K$ (see [18] for $p>2$ and [ 8 , Ch. XII, Th. 2.5 on pp. 244-245]).

Theorem 1.2 (Theorem 2.5 on pp. 244-245 of [8]). Assume that $p:=\operatorname{char}(K)>0$ and $K$ is finitely generated over the finite prime field $\mathbf{F}_{p}$.

Then for all abelian varieties $A$ and $B$ over $K$ and every prime $\ell \neq \operatorname{char}(K)$ the Galois module $V_{\ell}(A)$ is semisimple and the natural embedding of $\mathbf{Z}_{\ell}$-modules

$$
\operatorname{Hom}(A, B) \otimes \mathbf{Z}_{\ell} \hookrightarrow \operatorname{Hom}_{\mathrm{Gal}(K)}\left(T_{\ell}(A), T_{\ell}(B)\right)
$$

is bijective.

Remark 1.3. In fact, Theorem 1.2 follows even from a special case of Theorem 1.1 that deals only with principally polarized abelian varieties (i.e., when $d=1$ ), see Remark 3.12

By Lemma 1 of [16, Sect. 1] the second assertion of Theorem 1.2 implies the following statement.

Theorem 1.4. Assume that $p:=\operatorname{char}(K)>0$ and $K$ is finitely generated over the finite prime field $\mathbf{F}_{p}$.

Then for all abelian varieties $A$ and $B$ over $K$ the natural embedding of $\mathbf{Q}_{\ell \text {-vector }}$ spaces

$$
\operatorname{Hom}(A, B) \otimes \mathbf{Q}_{\ell} \hookrightarrow \operatorname{Hom}_{\mathrm{Gal}(K)}\left(V_{\ell}(A), V_{\ell}(B)\right)
$$

is bijective.

1.5. Let $K$ be a field that is finitely generated over the finite prime field $\mathbf{F}_{p}$ and $A$ an abelian variety of positive dimension over $K$. Let $\ell$ be a prime different from $p$. By Theorem 1.4 (applied to $B=A$ ) and Theorem 1.2, the $\operatorname{Gal}(K)$-module $V_{\ell}(A)$ is semisimple and

$$
\operatorname{End}_{\mathrm{Gal}(K)}\left(V_{\ell}(A)\right)=\operatorname{End}(A) \otimes \mathbf{Q}_{\ell}=\operatorname{End}^{0}(A) \otimes_{\mathbf{Q}} \mathbf{Q}_{\ell} .
$$


Since $G_{\ell, A, K}$ is the image of $\operatorname{Gal}(K) \rightarrow \operatorname{Aut}_{\mathbf{z}_{\ell}}\left(T_{\ell}(A)\right) \subset \operatorname{Aut}_{\mathbf{Q}_{\ell}}\left(V_{\ell}(A)\right)$, the $G_{\ell, A, K^{-}}$ module $V_{\ell}(A)$ is semisimple and

$$
\operatorname{End}_{G_{\ell, A, K}}\left(V_{\ell}(A)\right)=\operatorname{End}(A) \otimes \mathbf{Q}_{\ell}=\operatorname{End}^{0}(A) \otimes \mathbf{Q}_{\ell} \mathbf{Q}_{\ell}
$$

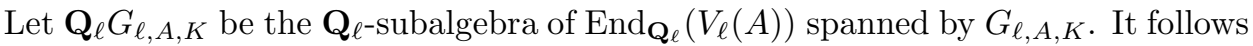
from the Jacobson density theorem [6, Ch. XVII, Sect. 3, Th. 1] that $\mathbf{Q}_{\ell} G_{\ell, A, K}$ coincides with the centralizer of $\operatorname{End}(A) \otimes \mathbf{Q}_{\ell}$ in $\operatorname{End}_{\mathbf{Q}_{\ell}}\left(V_{\ell}(A)\right)$. It follows easily

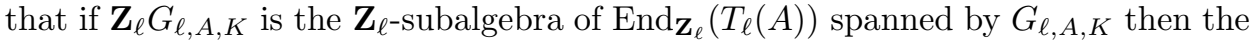
centralizer of $\operatorname{End}(A) \otimes \mathbf{Z}_{\ell}$ in $\operatorname{End}_{\mathbf{z}_{\ell}}\left(T_{\ell}(A)\right)$ contains $\mathbf{Z}_{\ell} G_{\ell, A, K}$ as a $\mathbf{Z}_{\ell \text {-submodule }}$ of finite index.

\section{MAin RESUlts}

The aim of this note is to prove variants of Theorem 1.2 where Tate modules are replaced by Galois modules $A_{n}$ and $B_{n}$. Most of our results were already proven in [19, 30] or stated in [22] under an additional assumption $p>2$. (See also [29] where the case of finite fields is discussed.)

Throughout this section, $K$ is a field that is finitely generated over the finite prime field $\mathbf{F}_{p}$.

Theorem 2.1. Let $A$ be an abelian variety of positive dimension over $K$. Then the set $\operatorname{Isog}(A, K)$ is finite.

Remark 2.2. A weaker version of Theorem 2.1 (where $\operatorname{Isog}(A, K)$ is replaced by its subset that consists of abelian varieties that admit a polarization of degree prime to $p$ ) is proven in [19, Th. 6.1] under an additional assumption that $p>2$.

Corollary 2.3. Let $A$ be an abelian variety of positive dimension over $K$. There exists a positive integer $r=r(A)$ that is not divisible by $p$ and enjoys the following properties.

(i) If $C$ is an abelian variety over $K$ that admits a $K$-isogeny $C \rightarrow A$ whose degree is not divisible by $p$ then there exists a $K$-isogeny $\beta: A \rightarrow C$ with $\operatorname{ker}(\beta) \subset A_{r}$.

(ii) If $n$ is a positive integer that is not divisible by $p$ and $W$ is a Galois submodule in $A_{n}$ then there exists $u \in \operatorname{End}(A)$ such that

$$
r W \subset u\left(A_{n}\right) \subset W .
$$

(iii) For all but finitely many primes $\ell$ the Galois module $A_{\ell}$ is semisimple.

Remark 2.4. Corollary 2.3(iii) is proven in [19, Th. 1.1] under an additional assumption $p>2$.

Theorem 2.5. Let $A$ be an abelian variety over $K$. Then there exists a positive integer $r_{1}=r_{1}(A)$ that enjoys the following properties.

Let $n$ be a positive integer that is not divisible by $p$ and $u_{n}$ an endomorphism of the Galois module $A_{n}$. If we put $m:=n /\left(n, r_{1}\right)$ then there exists $u \in \operatorname{End}(A)$ such that both $u$ and $u_{n}$ induce the same endomorphism of the Galois module $A_{m}$.

If $A$ and $B$ are abelian varieties over $K$ then applying Theorem 2.5 to their product $X=A \times B$, we obtain the following statement.

Theorem 2.6. Let $A$ and $B$ be abelian varieties over $K$. Then there exists a positive integer $r_{2}=r_{2}(A, B)$ that enjoys the following properties. 
Let $n$ be a positive integer that is not divisible by $p$ and $u_{n}: A_{n} \rightarrow B_{n}$ a homomorphism of the Galois modules. If we put $m:=n /\left(n, r_{2}\right)$ then there exists $u \in \operatorname{Hom}(A, B)$ such that both $u$ and $u_{n}$ induce the same homomorphism of the Galois modules $A_{m} \rightarrow B_{m}$.

Theorem 2.6 implies readily the following assertion.

Corollary 2.7. Let $A$ and $B$ be abelian varieties over $K$. Then for all but finitely many primes $\ell$ the natural injection

$$
\operatorname{Hom}(A, B) \otimes \mathbf{Z} / \ell \mathbf{Z} \hookrightarrow \operatorname{Hom}_{\mathrm{Gal}(K)}\left(A_{\ell}, B_{\ell}\right)
$$

is bijective.

Remark 2.8. Theorem 2.6 was stated without proof in 22$]$ under an additional condition that $p>2$. Corollary 2.7 was proven in [19, Th. 1.1] under an additional condition that $p>2$.

Theorem 2.9. Let $A$ be an abelian variety of positive dimension over $K$. Then for all but finitely many primes $\ell$ the centralizer of $\operatorname{End}(A) \otimes \mathbf{Z}_{\ell}$ in $\operatorname{End} \mathbf{z}_{\ell}\left(T_{\ell}(A)\right)$ coincides with $\mathbf{Z}_{\ell} G_{\ell, A, K}$.

Remark 2.10. When $K$ is a field of characteristic zero that is finitely generated over $\mathbf{Q}$, an analogue of Theorem 2.9 was proven by G. Faltings [3, Sect. 3, Th. $1(\mathrm{c})]$.

Recall that an old result of Grothendieck [10] asserts that in characteristic $p$ an abelian variety of CM-type is isogenous to an abelian variety that is defined over a finite field. (The converse follows from a theorem of Tate [16.)

Theorem 2.11. Let $X$ be an abelian variety over $K$. Suppose that for infinitely many primes $\ell$ the group $\tilde{G}_{\ell, X, K}$ is commutative. Then $X$ is an abelian variety of $C M$ type over $K$ and therefore is isogenous over $\bar{K}$ to an abelian variety that is defined over a finite field.

Theorem 2.11 may be strengthened as follows.

Theorem 2.12. Let $X$ be an abelian variety over $K$. Suppose that for infinitely many primes $\ell$ the group $\tilde{G}_{\ell, X, K}$ is $\ell$-solvable, i.e., its Jordan-Hölder factors are either $\ell$-groups or groups whose order is not divisible by $\ell$. Then there is a finite Galois extension $K^{\prime} / K$ such that $X \times_{K} K^{\prime}$ is an abelian variety of CM type over $K^{\prime}$ and therefore is isogenous over $\bar{K}$ to an abelian variety that is defined over a finite field.

Theorem 2.12 combined with the celebrated theorem of Feit-Thompson (about solvability of groups of odd order) implies readily the following statement.

Corollary 2.13. Let $X$ be an abelian variety of positive dimension over $K$ that is not isogenous over $\bar{K}$ to an abelian variety that is defined over a finite field. Then for all but finitely many primes $\ell$ the group $\tilde{G}_{\ell, X, K}$ is not solvable and its order is divisible by $2 \ell$.

Remark 2.14. See [26, 28, 27] for plenty of explicit examples of abelian varieties in characteristic $p$ without CM.

Theorem 2.11] was proven in [20] under an additional assumption that $p>2$. Theorem 2.12 was stated without proof in 22] under an additional assumption that $K$ is global and $p>2$. 
In order to state another (partial) strenghening of Theorem 2.11 we need to introduce the following notation. If $\mathcal{A}$ a commutative group then we write $\operatorname{TORS}(\mathcal{A})$ for its subgroup of all periodic elements and $\operatorname{TORS}(\mathcal{A})($ non $-p)$ that consists of all elements of $\operatorname{TORS}(\mathcal{A})$, whose order is prime to $p$.

Theorem 2.15. Let $K^{a b} \subset \bar{K}_{s}$ be the maximal abelian extension of $K$. Let $X$ be a simple abelian variety over $K$. If $\operatorname{TORS}\left(X\left(K^{a b}\right)\right)($ non $-p)$ is infinite then $X$ is an abelian variety of $C M$-type over $K$ and therefore is isogenous over $\bar{K}$ to an abelian variety that is defined over a finite field.

Remark 2.16. In characteristic zero an analogue of Theorem 2.15 was proven in [24, Th. 1.5].

Theorem 2.15implies readily the following statement. (Compare with 24, Corollary on p. 132].)

Corollary 2.17. Let $K^{a b} \subset \bar{K}_{s}$ be the maximal abelian extension of $K$. Let $X$ be an abelian variety of positive dimension over $K$. Let $X_{1}, \ldots, X_{r}$ be simple abelian varieties over $K$ such that the product $\prod_{i=1}^{r} X_{i}$ is $K$-isogenous to $X$. Then $\operatorname{TORS}\left(X\left(K^{a b}\right)\right)($ non $-p)$ is finite if and only if all the groups $\operatorname{TORS}\left(X_{i}\left(K^{a b}\right)\right)$ (non$p)$ are finite, i.e., all $X_{i}$ are not of CM-type over $K(1 \leq i \leq r)$.

Now we discuss the torsion of abelian varieties in infinite Galois extensions of $K$ with finite "field of constants".

Theorem 2.18. Let $X$ be an abelian variety of positive dimension over $K$ such that the center of $\operatorname{End}^{0}(X)$ is a direct sum of totally real number fields. Let $K^{\prime} \subset \bar{K}_{s}$ be an infinite Galois extension of $K$. Let $\mathbf{F}^{\prime}$ be the algebraic closure of $\mathbf{F}_{p}$ in $K^{\prime}$ and suppose that $\mathbf{F}^{\prime}$ is a finite field. Then $\operatorname{TORS}\left(X\left(K^{\prime}\right)\right)($ non $-p)$ is finite.

Theorem 2.18 is an immediate corollary of the conjunction of following two assertions.

Theorem 2.19. Let $X$ be an abelian variety of positive dimension over $K$ such that the center of $\operatorname{End}^{0}(X)$ is a direct sum of totally real number fields. Let $K^{\prime} \subset \bar{K}_{s}$ be an infinite Galois extension of $K$. If $\ell \neq p$ is a prime such that the $\ell$-primary component of $\operatorname{TORS}\left(X\left(K^{\prime}\right)\right)$ is infinite then $K^{\prime}$ contains all $\ell$-power roots of unity. In particular, the algebraic closure of $\mathbf{F}_{p}$ in $K^{\prime}$ is infinite.

Theorem 2.20. Let $X$ be an abelian variety of positive dimension over $K$ such that the center of $\operatorname{End}^{0}(X)$ is a direct sum of totally real number fields. Let us choose a polarization $\lambda: X \rightarrow X^{t}$ that is defined over $K$. Let $\ell \neq p$ be a prime that enjoys the following properties:

(i) $\ell$ is odd and prime to $\operatorname{deg}(\lambda)$;

(ii) The $\operatorname{Gal}(K)$-module $X_{\ell}$ is semisimple and

$$
\operatorname{End}_{\mathrm{Gal}(K)}\left(X_{\ell}\right)=\operatorname{End}(X) \otimes \mathbf{Z} / \ell \mathbf{Z} .
$$

(iii) If $C$ is the center of $\operatorname{End}(X)$ then $C / \ell C$ is the center of $\operatorname{End}(X) / \ell \operatorname{End}(X)$. If the $\ell$-primary component of $\operatorname{TORS}\left(X\left(K^{\prime}\right)\right)$ does not vanish then $K^{\prime}$ contains a primitive $\ell$ th root of unity. In particular, if $\mathbf{F}^{\prime}$ is the algebraic closure of $\mathbf{F}_{p}$ in $K^{\prime}$ then its order is strictly greater than $\ell$.

Remark 2.21. Let $S$ be the set of primes $\ell$ that do not enjoy either property (i) or property (ii). Then $S$ is finite. 
The paper is organized as follows. In Section 3 we discuss isogenies of abelian varieties and their kernels (viewed as finite Galois modules). One of the goals of our approach is to stress the role of analogues of Tate's finiteness conjecture for isogeny classes of abelian varieties. In Section 4 we prove all the main results except Theorem 2.12, which will be proven in Section 5. Section 6] contains additional references to results that may be extended to characteristic 2 case.

Acknowledgements. I am grateful to Alexey Parshin, Chad Schoen and Doug Ulmer for their interest in this paper. My special thanks go to the referees, whose comments helped to improve the exposition.

The final version of this paper was prepared during my stay at Max-PlanckInstitut für Mathematik (Bonn) in September 2013: I am grateful to the MPI for the hospitality and support.

\section{Isogenies AND Finite Galois modules}

We write $\mathbf{P}$ for the set of all primes. Let $K$ be a field. Let $P \subset \mathbf{P}$ be a nonempty set of primes that does not contain $\operatorname{char}(K)$. If $X$ and $Y$ are abelian varieties over $K$ then a $K$-isogeny $X \rightarrow Y$ is called a $P$-isogeny if all prime divisors of its degree are elements of $P$. For example, if $P$ is a singleton $\{\ell\}$ then a $P$-isogeny is nothing else but an $\ell$-power isogeny. We say that $X$ and $Y$ are $P$-isogenous over $K$ if there is a $P$-isogeny $X \rightarrow Y$ that is defined over $K$. The property to be $P$-isogenous is an equivalence relation. Indeed, one has only to check that there exists a $P$ isogeny $v: Y \rightarrow X$ that is defined over $K$. Indeed, thanks to Lagrange theorem, $\operatorname{ker}(u) \subset X_{n}$ where

$$
n:=\operatorname{deg}(u)=\#(\operatorname{ker}(u)) .
$$

It follows that there is a $K$-isogeny $v: Y \rightarrow X$ such that the composition $v u: X \rightarrow$ $Y \rightarrow X$ coincides with multiplication by $n$ in $X$. This implies that

$$
n^{2 \operatorname{dim}(X)}=\operatorname{deg}(v u)=\operatorname{deg}(v) \operatorname{deg}(u) .
$$

Since $u$ is a $P$-isogeny, all prime divisors of $n$ belong to $P$. This implies that all prime divisors of $\operatorname{deg}(v)$ also belong to $P$, i.e., $v$ is a $P$-isogeny and we are done.

Let $X^{t}$ and $Y^{t}$ be the dual abelian varieties (over $K$ ) of $X$ and $Y$ respectively and

$$
u^{t}: Y^{t} \rightarrow X^{t}, v^{t}: X^{t} \rightarrow Y^{t}
$$

be the $K$-isogenies that are duals of $u$ and $v$ respectively. Since

$$
\operatorname{deg}\left(u^{t}\right)=\operatorname{deg}(u) \cdot \operatorname{deg}\left(v^{t}\right)=\operatorname{deg}(v),
$$

$X^{t}$ and $Y^{t}$ are also $P$-isogenous over $K$. (Warning: $X$ and $X^{t}$ are not necessarily $P$-isogenous!) This implies that if $X$ and $Y$ are $P$-isogenous over $K$ then $\left(X \times X^{t}\right)^{4}$ and $\left(Y \times Y^{t}\right)^{4}$ are also $P$-isogenous over $K$.

We write $\operatorname{Is}_{P}(X, K)$ for the set of isomorphism classes of abelian varieties $Y$ over $K$ that are $P$-isogenous to $X$ over $K$. We write $\operatorname{Is}_{P}(X, K, 1)$ for the subset of $\operatorname{Isog}_{P}(X, K)$ that consists of all isomorphism classes of $Y$ with principal polarization over $K$. For example, if $P$ is $\mathbf{P} \backslash\{\operatorname{char}(K)\}$ then

$$
\operatorname{Is}_{P}(X, K)=\operatorname{Isog}(X, K), \operatorname{Is}_{P}(X, K, 1)=\operatorname{Isog}(X, K, 1) .
$$

Now Theorem 2.1 becomes an immediate corollary of Theorem 1.1 and the following statement. 
Theorem 3.1. Let $X$ be an abelian variety over a field $K$. Suppose that the set $\operatorname{Is}_{P}\left(\left(X \times X^{t}\right)^{4}, K, 1\right)$ is finite. Then the set $\operatorname{Is}_{P}(X, K)$ is also finite.

Proof. (i) Let us fix an abelian variety $X$ be over $K$. Let $Y$ be an abelian variety over $K$ that is $P$-isogenous to $X$ over $K$. As we have seen, $\left(Y \times Y^{t}\right)^{4}$ is $P$-isogenous to $\left(X \times X^{t}\right)^{4}$ over $K$. Recall [19, 23, 8] (see also [29, Sect. 7]) that $\left(Y \times Y^{t}\right)^{4}$ admits a principal polarization over $K{ }^{1}$. Since the set $\operatorname{Isog}_{P}\left(\left(Y \times Y^{t}\right)^{4}, K, 1\right)$ is finite, the set of $K$-isomorphism classes of all $\left(Y \times Y^{t}\right)^{4}$ (with fixed $X$ ) is finite. On the other hand, each $Y$ is isomorphic to a $K$-abelian subvariety of $\left(Y \times Y^{t}\right)^{4}$ over $K$. But the set of isomorphism classes of abelian subvarieties of a given abelian variety is finite [7. This implies that the set of $K$-isomorphism classes of all $Y$ 's is finite.

Corollary 3.2. Let $X$ be an abelian variety of positive dimension over a field $K$. Suppose that the set $\operatorname{Is}_{P}\left(\left(X \times X^{t}\right)^{4}, K, 1\right)$ is finite.

Then there exists a positive integer $r=r(X)$ that is not divisible by $\operatorname{char}(K)$ and enjoys the following properties.

(i) If $Y$ is an abelian variety over $K$ that is $P$-isogenous to $X$ over $K$ then there exist a $P$-isogeny $\beta: X \rightarrow Y$ over $K$ with $\operatorname{ker}(\beta) \subset X_{r}$.

(ii) If $n$ is a positive integer, all whose prime divisors lie in $P$ and $W$ is a Galois submodule in $X_{n}$, then there exists $u \in \operatorname{End}(X)$ such that

$$
r W \subset u\left(X_{n}\right) \subset W .
$$

Proof. By Theorem 3.1, there are finitely many $K$-abelian varieties $Y_{1}, \ldots, Y_{d}$ that are $P$-isogenous to $X$ over $K$ and such that every $K$-abelian variety $Y$ that is $P$ isogenous to $X$ over $K$ is $K$-isomorphic to one of $Y_{j}$. For each $Y_{i}$ pick a $P$-isogeny $\beta_{i}: X \rightarrow Y_{i}$ that is defined over $K$. Clearly, $\operatorname{ker}\left(\beta_{i}\right) \subset X_{m_{i}}$ where $m_{i}=\operatorname{deg}\left(\beta_{i}\right)$. Let us put $r=\prod_{i=1}^{d} m_{i}$. Clearly, for all $Y_{i}$

$$
\operatorname{ker}\left(\beta_{i}\right) \subset X_{m_{i}} \subset X_{r} .
$$

This implies that for every $K$-abelian variety $Y$ that is $P$-isogenous to $X$ over $K$ there exists a $P$-isogeny $\beta: X \rightarrow Y$ over $K$ whose kernel lies in $X_{r}$. This proves (i), since every prime divisor of $r$ is a prime divisor of one of $m_{i}=\operatorname{deg}\left(\beta_{i}\right)$ and therefore lies in $P$.

Proof of (ii) The quotient $Y=X / W$ is an abelian variety over $K$. The canonical map $\pi: X \rightarrow X / W=Y$ is a $P$-isogeny over $K$, because $\operatorname{deg}(\pi)=\#(W)$ divides $\#\left(X_{n}\right)=n^{2 \operatorname{dim}(X)}$. This implies that $Y$ is $P$-isogenous to $X$ over $K$.

The rest of the proof goes literally (with the same notation) as in [29, Sect. 8, pp. 331-332] provided one replaces the reference to [29, Cor. 3.5(i)] by the already proven case (i) of Corollary 3.2. (In [29, $n_{X}: X \rightarrow X$ and $n_{Y}: Y \rightarrow Y$ denote the multiplication by $n$ in $X$ and $Y$ respectively.)

Theorem 3.3. Suppose that $P$ is infinite. Let $X$ be an abelian variety of positive dimension over a field $K$. Suppose that the set $\operatorname{Is}_{P}\left(\left(X \times X^{t}\right)^{4}, K, 1\right)$ is finite.

Then for all but finitely many primes $\ell \in P$ the Galois module $X_{\ell}$ enjoys the following properties.

If $W$ is a Galois submodule in $X_{\ell}$ then there exists $\tilde{u} \in \operatorname{End}(X) \otimes \mathbf{Z} / \ell Z$ such that $\tilde{u}^{2}=\tilde{u}$ and $\tilde{u}\left(X_{\ell}\right)=W$. In particular, the Galois module $X_{n}$ splits into a direct sum

$$
X_{\ell}=\tilde{u}\left(X_{\ell}\right) \oplus(1-\tilde{u})\left(X_{\ell}\right)=W \oplus(1-\tilde{u})\left(X_{\ell}\right)
$$

${ }^{1}$ In [8. Ch. IX, Sect. 1] Deligne's proof is given. 
of its Galois submodules $W$ and $(1-\tilde{u})\left(X_{\ell}\right)$.

Theorem 3.3 implies immediately the following assertion.

Corollary 3.4. Suppose that $P$ is infinite. Let $X$ be an abelian variety of positive dimension over a field $K$. Suppose that the set $\operatorname{Is}_{P}\left(\left(X \times X^{t}\right)^{4}, K, 1\right)$ is finite.

Then for all but finitely many primes $\ell \in P$ the Galois module $X_{\ell}$ is semisimple.

Proof of Theorem 3.3. It is well known that for all but finitely many primes $\ell$ the finite-dimensional $\mathbf{F}_{\ell}$-algebra $\operatorname{End}(X) \otimes \mathbf{Z} / \ell \mathbf{Z}$ is semisimple. (See, e.g., 19, Lemma 3.2].) Let $r$ be as in Corollary 3.2. Now let $\ell \in P$ be a prime that does not divide $r$ and such that $\operatorname{End}(X) \otimes \mathbf{Z} / \ell \mathbf{Z}$ is semisimple. Let $W$ be a Galois submodule in $X_{\ell}$. By Corollary 3.2 there exists $u \in \operatorname{End}(X)$ such that

$$
r W \subset u\left(X_{\ell}\right) \subset W .
$$

Since $\ell$ does not divide $r$, we have $r W=W$ and therefore $u\left(X_{\ell}\right)=W$. Let $u_{\ell}$ be the image of $u$ in $\operatorname{End}(X) \otimes \mathbf{Z} / \ell \mathbf{Z}$. Clearly,

$$
u_{\ell}\left(X_{\ell}\right)=u\left(X_{\ell}\right)=W
$$

Let $I$ be the right ideal in semisimple $\operatorname{End}(X) \otimes \mathbf{Z} / \ell \mathbf{Z}$ generated by $u_{\ell}$. The semisimplicity implies that there exists an idempotent $\tilde{u}$ that generates $I$. It follows that

$$
W=u_{\ell}\left(X_{\ell}\right)=\tilde{u}\left(X_{\ell}\right)
$$

We will need the following lemma [29, Lemma 9.2 on p. 333].

Lemma 3.5. Let $Y$ be an abelian variety of positive dimension over an arbitrary field $K$. Then there exists a positive integer $h=h(Y, K)$ that enjoys the following properties.

If $n$ is a positive integer that is not divisible by $\operatorname{char}(K), u, v \in \operatorname{End}(Y)$ are endomorphisms of $Y$ such that

$$
\left\{\operatorname{ker}(u) \bigcap Y_{n}\right\} \subset\left\{\operatorname{ker}(v) \bigcap Y_{n}\right\}
$$

then there exists a $K$-isogeny $w: Y \rightarrow Y$ such that

$$
h v-w u \in n \cdot \operatorname{End}(Y) .
$$

In particular, the images of $h v$ and $w u$ in

$$
\operatorname{End}(Y) \otimes \mathbf{Z} / n \mathbf{Z} \subset \operatorname{End}_{\operatorname{Gal}(K)}\left(Y_{n}\right) \subset \operatorname{End}_{\mathbf{Z} / n \mathbf{Z}}\left(Y_{n}\right)
$$

coincide.

Theorem 3.6. Let $X$ be an abelian variety of positive dimension over a field $K$. Suppose that the set $\operatorname{Is}_{P}\left(\left(X \times X^{t}\right)^{4}, K, 1\right)$ is finite. Then there exists a positive integer $r_{1}=r_{1}(X, K)$ that enjoys the following properties.

Let $n$ be a positive integer, all whose prime divisors lie in $P$ and $m=n /\left(n, r_{1}\right)$. If $u_{n} \in \operatorname{End}_{\operatorname{Gal}(K)}\left(X_{n}\right)$ then there exists $u \in \operatorname{End}(X)$ such that the images of $u_{n}$ and $u$ in $\operatorname{End}_{\operatorname{Gal}(K)}\left(X_{m}\right)$ coincide.

Proof. Let us put $Y=X \times X$. Then $\left(Y \times Y^{t}\right)^{4}=\left(X \times X^{t}\right)^{8}$. Let $r(Y)$ be as in Corollary 3.2 and $h(Y)$ as in Lemma 3.5 Let us put

$$
r_{1}=r_{1}(X, K)=r(Y, K) h(Y, K) \text {. }
$$


Now the proof goes literally as the the proof of Theorem 4.1 in [29, Sect. 10], provided one replaces the references to Cor. 3.5 and Lemma 9.2 of 29 by references to Cor. 3.2 and Lemma 3.5 respectively.

Let $A$ and $B$ be abelian varieties over $K$. Applying Theorem to $X=A \times B$ and using the obvious compatible decompositions

$$
\begin{gathered}
\operatorname{End}(X)=\operatorname{End}(A) \oplus \operatorname{End}(B) \oplus \operatorname{Hom}(A, B) \oplus \operatorname{Hom}(B, A), \\
\operatorname{End}_{\mathrm{Gal}(K)}\left(X_{n}\right)=
\end{gathered}
$$

$\operatorname{End}_{\mathrm{Gal}(K)}\left(A_{n}\right) \oplus \operatorname{End}_{\mathrm{Gal}(K)}\left(B_{n}\right) \oplus \operatorname{Hom}_{\mathrm{Gal}(K)}\left(A_{n}, B_{n}\right) \oplus \operatorname{Hom}_{\mathrm{Gal}(K)}\left(B_{n}, A_{n}\right)$, we obtain the following statement.

Theorem 3.7. Let $A$ and $B$ be abelian varieties of positive dimension over a field $K$. Suppose that the set $\operatorname{Isog}_{P}\left(\left(A \times B \times A^{t} \times B^{t}\right)^{8}, K, 1\right)$ is finite. Then there exists a positive integer $r_{2}=r_{2}(A, B, K)=r_{1}(A \times B, K)$ that enjoys the following properties.

Let $n$ be a positive integer, all whose prime divisors lie in $P$ and $m=n /\left(n, r_{1}\right)$. If $u_{n} \in \operatorname{Hom}_{\operatorname{Gal}(K)}\left(A_{n}, B_{n}\right)$ then there exists $u \in \operatorname{Hom}(A, B)$ such that the images of $u_{n}$ and $u$ in $\operatorname{Hom}_{\operatorname{Gal}(K)}\left(A_{m}, B_{m}\right)$ coincide.

Corollary 3.8. Let $A$ and $B$ be abelian varieties of positive dimension over a field $K$. Suppose that the set $\operatorname{Isog}_{P}\left(\left(A \times B \times A^{t} \times B^{t}\right)^{8}, K, 1\right)$ is finite. Then for all primes $\ell \in P$ the natural injection

$$
\operatorname{Hom}(A, B) \otimes \mathbf{Z}_{\ell} \hookrightarrow \operatorname{Hom}_{\mathrm{Gal}(K)}\left(T_{\ell}(A), T_{\ell}(B)\right)
$$

is bijective.

Proof of Corollary 3.8. Let $r_{2}=r_{2}(A, B)$ be as in Theorem 3.7. Let $\ell^{i_{0}}$ be the exact power of $\ell$ that divides $r_{2}$. Let $v \in \operatorname{Hom}_{\operatorname{Gal}(K)}\left(T_{\ell}(A), T_{\ell}(B)\right)$. For each $i>i_{0}, v$ induces a homomorphism $v_{i} \in \operatorname{Hom}_{\operatorname{Gal}(K)}\left(A_{\ell^{i}}, B_{\ell^{i}}\right)$. By Theorem 3.7, there exists $u_{i} \in \operatorname{Hom}(A, B)$ such that the images of $u_{i}$ and $v_{i}$ in $\operatorname{Hom}\left(A_{\ell^{i-i_{0}}}, B_{\ell^{i-i_{0}}}\right)$ coincide. This means that $u_{i}-v$ sends $T_{\ell}(A)$ into $\ell^{i-i_{0}} T_{\ell}(B)$. It follows that $v$ coincides with the limit of the sequence $\left\{u_{i}\right\}_{i>i_{0}}^{\infty}$ in $\operatorname{Hom}_{\mathbf{Z}_{\ell}}\left(T_{\ell}(A), T_{\ell}(B)\right)$ with respect to $\ell$-adic topology. Since $\operatorname{Hom}(A, B) \otimes \mathbf{Z}_{\ell}$ is a compact and therefore a closed subset of $\operatorname{Hom}_{\mathbf{Z}_{\ell}}\left(T_{\ell}(A), T_{\ell}(B)\right)$, the limit $v$ also lies in $\operatorname{Hom}(A, B) \otimes \mathbf{Z}_{\ell}$.

The following lemma will be proven at the end of this section.

Lemma 3.9. Let $X$ be an abelian variety of positive dimension over a field $K$. Suppose that the set $\operatorname{Is}_{P}\left(\left(X \times X^{t}\right)^{4}, K, 1\right)$ is finite. Let $r=r(X, K)$ be as in Corollary 3.2. Then every $\ell \in P$ enjoys the following properties.

Let $\mathbf{S}$ be a Galois-invariant $\mathbf{Z}_{\ell}$-submodule in $T_{\ell}(X)$ such that the quotient $T_{\ell}(X) / \mathbf{S}$ is torsion-free. Then there exists $u \in \operatorname{End}(X) \otimes \mathbf{Z}_{\ell}$ such that

$$
r_{1} \cdot \mathbf{S} \subset u\left(T_{\ell}(X)\right) \subset \mathbf{S} .
$$

Theorem 3.10. Let $X$ be an abelian variety of positive dimension over a field $K$. Suppose that the set $\operatorname{Is}_{P}\left(\left(X \times X^{t}\right)^{8}, K, 1\right)$ is finite. Then every $\ell \in P$ enjoys the following properties.

If $\mathbf{W}$ is a $\mathrm{Gal}(K)$-invariant $\mathbf{Q}_{\ell}$-vector subspace in $V_{\ell}(X)$ then there exists $\tilde{u} \in$ $\operatorname{End}(X) \otimes \mathbf{Q}_{\ell}$ such that $\tilde{u}^{2}=\tilde{u}$ and $\tilde{u}\left(V_{\ell}(X)\right)=\mathbf{W}$. In particular, $V_{\ell}(X)$ splits into a direct sum

$$
V_{\ell}(X)=\tilde{u}\left(V_{\ell}(X)\right) \oplus(1-\tilde{u})\left(V_{\ell}(X)\right)=\mathbf{W} \oplus(1-\tilde{u})\left(V_{\ell}(X)\right)
$$


of its Galois submodules $\mathbf{W}$ and $(1-\tilde{u})\left(V_{\ell}(X)\right)$ and the $\operatorname{Gal}(K)$-module $V_{\ell}(X)$ is semisimple.

Proof of Theorem 3.10. Let us put $\mathbf{S}=\mathbf{W} \cap T_{\ell}(X)$. Clearly, $\mathbf{S}$ is a Galois-invariant

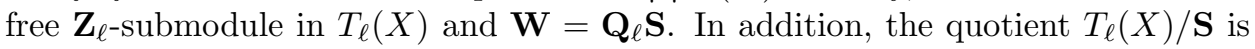
torsion-free.

By Lemma 3.9. there exists $u \in \operatorname{End}(X) \otimes \mathbf{Z}_{\ell}$ such that

$$
r \cdot \mathbf{S} \subset u\left(T_{\ell}(X)\right) \subset \mathbf{S} .
$$

It follows that

$$
r \cdot \mathbf{W} \subset u\left(V_{\ell}(X)\right) \subset \mathbf{W} .
$$

Since $r \cdot \mathbf{W}=\mathbf{W}$, we have $u\left(V_{\ell}(X)\right)=\mathbf{W}$. Notice that

$$
\operatorname{End}(X) \otimes \mathbf{Z}_{\ell} \subset \operatorname{End}(X) \otimes \mathbf{Q}_{\ell}=\operatorname{End}^{0}(X) \otimes_{\mathbf{Q}} \mathbf{Q}_{\ell}
$$

and $\operatorname{End}^{0}(X)$ is a finite-dimensional semisimple $\mathbf{Q}$-algebra. It follows that $\operatorname{End}(X) \otimes$

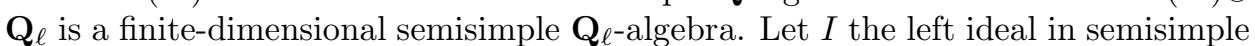
$\operatorname{End}(X) \otimes \mathbf{Q}_{\ell}$ generated by $u$; there is an idempotent $\tilde{u}$ that generates $I$. Clearly.

$$
\tilde{u}\left(V_{\ell}(X)\right)=u\left(V_{\ell}(X)\right)=\mathbf{W} .
$$

Proof of Lemma 3.9. If $S S=\{0\}$ then we just put $u=0$. If $\mathbf{S}=T_{\ell}(X)$ then we take as $u$ the identity automorphism $1_{X}$ of $X$.

So, further we assume that $S S$ is a proper free $\mathbf{Z}_{\ell}$-module in $T_{\ell}(X)$ of positive rank say, $d$ and let $\left\{e_{1}, \ldots, e_{j} \ldots e_{d}\right\}$ be its basis. Since $S S$ is pure in $T_{\ell}(X)$, for all positive integers $i$ the natural homomorphism of Galois modules

$$
S S_{i}:=S S / \ell^{i} S S \rightarrow T_{\ell}(X) / \ell^{i} T_{\ell}(X)=X_{\ell^{i}}
$$

is an injection of free $\mathbf{Z} / \ell^{i} \mathbf{Z}$-modules. Further, we will identify $S S_{i}$ with its image in $X_{\ell^{i}}$. We write $\bar{e}_{j}^{i}$ for the image of $e_{j}$ in $\mathbf{S}_{i} \subset X_{\ell^{i}}$; clearly, the $d$-element set $\left\{\bar{e}_{j}^{i}\right\}_{j=1}^{d}$ is a basis of the free $\mathbf{Z} / \ell^{i} \mathbf{Z}$-module $\mathbf{S}_{i}$. By Corollary 3.2 applied to $n=\ell^{i}$ and $W=S S_{i}$ there exists $u_{i} \in \operatorname{End}(X)$ such that

$$
r S S_{i} \subset u_{i}\left(X_{\ell^{i}}\right) \subset S S_{i} .
$$

In particular, $u_{i}\left(X_{\ell^{i}}\right)$ contains $r \bar{e}_{j}^{i}$ for all $j$. It is also clear that for each $z \in T_{\ell}(X)$

$$
u_{i}(z) \in S S+\ell^{i} T_{\ell}(X) .
$$

For each $\bar{e}_{j}^{i}$ pick an element

$$
\bar{z}_{j}^{i} \in T_{\ell}(X) / \ell^{i} T_{\ell}(X)=X_{\ell^{i}}
$$

such that $u_{i}\left(\bar{z}_{j}^{i}\right)=r \bar{e}_{j}^{i}$. Let us pick $z_{j}^{i} \in T_{\ell}(X)$ such that its image in $X_{\ell^{i}}$ coincides with $\bar{z}_{j}^{i}$. Clearly, the image of $u_{i}\left(z_{j}^{i}\right)$ in $T_{\ell}(X) / \ell^{i} T_{\ell}(X)=X_{\ell^{i}}$ equals $r \bar{e}_{j}^{i}$. Using the compactness of $\operatorname{End}(X) \otimes \mathbf{Z}_{\ell}$ and $T_{\ell}(X)$, let us choose an infinite increasing sequence of positive integers $i_{1}<i_{2}<\cdots<i_{m}<\ldots$ such that $\left\{u_{i_{m}}\right\}_{m=1}^{\infty}$ converges in $\operatorname{End}(X) \otimes \mathbf{Z}_{\ell}$ to some $u$ and $\left\{z_{i_{m}}^{j}\right\}_{m=1}^{\infty}$ converges in $T_{\ell}(X)$ to some $z^{j}$ for all $j$ with $1 \leq j \leq d$. It follows that

$$
u\left(z^{j}\right)=\lim u_{i_{m}}\left(z_{i_{m}}^{j}\right)=r e_{j} .
$$

This implies that $u\left(T_{\ell}(X)\right) \supset r \cdot \mathbf{S}$. On the other hand, for each $z \in T_{\ell}(X)$

$$
u_{i_{m}}(z) \in \mathbf{S}+\ell^{i_{m}} T_{\ell}(X) .
$$


Since $\left\{i_{m}\right\}_{m=1}^{\infty}$ is an increasing set of positive integers, $\left\{S S+\ell^{i_{m}} T_{\ell}(X)\right\}_{m=1}^{\infty}$ is a decreasing set of compact sets whose intersection is compact $S S$. It follows that $u(z)=\lim u_{i_{m}}(z)$ lies in $S S$.

Lemma 3.11. Let $X$ be an abelian variety of positive dimension over $K$. Let $\ell$ be a prime that is different from $\operatorname{char}(K)$ and such that the $\operatorname{Gal}(K)$-module $X_{\ell}$ is semisimple and

$$
\operatorname{End}_{\mathrm{Gal}(K)}\left(X_{\ell}\right)=\operatorname{End}(X) \otimes \mathbf{Z} / \ell \mathbf{Z}
$$

Then the centralizer of $\operatorname{End}(X) \otimes \mathbf{Z}_{\ell}$ in $\operatorname{End}_{\mathbf{Z}_{\ell}}\left(T_{\ell}(X)\right)$ coincides with $\mathbf{Z}_{\ell} G_{\ell, X, K}$.

Proof of Lemma 3.11. Clearly, $\tilde{G}_{X, \ell, K}$ is the image of $\operatorname{Gal}(K) \rightarrow G_{\ell, X, K} \rightarrow \operatorname{Aut}_{\mathbf{F}_{\ell}}\left(X_{\ell}\right)$. It follows that the $\tilde{G}_{X, \ell, K}$-module $X_{\ell}$ is semisimple and

$$
\operatorname{End}_{\tilde{G}_{X, \ell, K}}\left(X_{\ell}\right)=\operatorname{End}(X) \otimes \mathbf{Z} / \ell \mathbf{Z} .
$$

By the Jacobson density theorem, $\mathbf{F}_{\ell} \tilde{G}_{X, \ell, K}$ coincides with the centralizer of $\operatorname{End}(X) \otimes$ $\mathbf{Z} / \ell \mathbf{Z}$ in $\operatorname{End}_{\mathbf{F}_{\ell}}\left(X_{\ell}\right)$. (Here $\mathbf{F}_{\ell} \tilde{G}_{X, \ell, K}$ is the $\mathbf{F}_{\ell}$-subalgebra of $\operatorname{End}_{\mathbf{F}_{\ell}}\left(X_{\ell}\right)$ spanned by $\tilde{G}_{X, \ell, K}$.)

Let $M$ be the centralizer of $\operatorname{End}(X) \otimes \mathbf{Z}_{\ell}$ in $\operatorname{End}_{\mathbf{z}_{\ell}}\left(T_{\ell}(X)\right)$. Clearly, $M$ is a

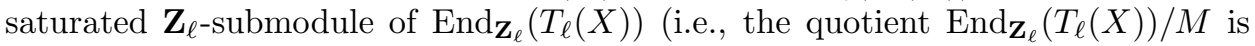
torsion-free); in addition, $M$ contains $\mathbf{Z}_{\ell} G_{\ell, X, K}$. We have

$$
M / \ell M \subset \operatorname{End}_{\mathbf{Z}_{\ell}}\left(T_{\ell}(X)\right) \otimes \mathbf{Z}_{\ell} / \ell \mathbf{Z}_{\ell}=\operatorname{End}_{\mathbf{F}_{\ell}}\left(X_{\ell}\right) .
$$

Clearly, $M / \ell M$ lies in the centralizer of

$$
\operatorname{End}(X) \otimes \mathbf{Z}_{\ell}=\otimes \mathbf{Z}_{\ell} / \ell \mathbf{Z}_{\ell}=\operatorname{End}(X) \otimes \mathbf{Z} / \ell \mathbf{Z} .
$$

This implies that

$$
M / \ell M \subset \mathbf{F}_{\ell} \tilde{G}_{X, \ell, K} \subset \operatorname{End}_{\mathbf{F}_{\ell}}\left(X_{\ell}\right) .
$$

On the other hand, the image of $\mathbf{Z}_{\ell} G_{\ell, X, K}$ in

$$
\operatorname{End}_{\mathbf{z}_{\ell}}\left(T_{\ell}(X)\right) \otimes \mathbf{Z}_{\ell} / \ell \mathbf{Z}_{\ell}=\operatorname{End}_{\mathbf{F}_{\ell}}\left(X_{\ell}\right)
$$

obviously coincides with $\mathbf{F}_{\ell} \tilde{G}_{X, \ell, K}$. Since this image lies in $M / \ell M$, we conclude that $M / \ell=\mathbf{F}_{\ell} \tilde{G}_{X, \ell, K}$ and $M=\mathbf{Z}_{\ell} G_{\ell, X, K}+\ell \cdot M$. It follows from Nakayama's

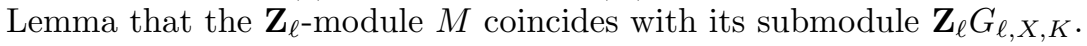

Remark 3.12. Let $P$ be a singleton $\{\ell\}$ and $d=1$. Now Theorem 1.1 combined with Corollary 3.8 and Theorem 3.10 implies readily Theorem 1.2 .

\section{Proof of main Results}

Throughout this section, $K$ is a field that is finitely generated over $\mathbf{F}_{p}$. Let us put $P=\mathbf{P} \backslash\{p\}$.

Proof of Corollary 2.3 and Theorem 2.5. Corollary 2.3 follows readily from Corollary 3.2 combined with Theorem 1.1. Theorem 2.5 follows readily from Theorem 3.6 combined with Theorem 1.1.

Proof of Theorems 2.1 and 2.6. One has only to combine Theorem 1.1 with Theorems 3.1 and 3.7 respectively.

Proof of Theorem 2.9. The assertion follows readily from Lemma 3.11 combined with Corollary 2.3 and Theorem 2.5. 
Proof of Theorem [2.11, The proof of [20, Theorem 4.7.4] works literally provided one replaces the reference to [19, Theorem 1.1.1] by references to Corollaries 2.3 and 2.7 .

Proof of Theorem 2.15. Theorem 2.15 is an immediate corollary of the conjunction of two following statements. (Compare with Theorems 2 and 3 on p. 133 of [24.)

Theorem 4.1. Let $X$ be a simple abelian variety over $K$ that is not of CM type. Let $\ell \neq p$ be a prime, $W$ a nonzero Galois-invariant $\mathbf{Q}_{\ell}$-vector space in $V_{\ell}(X)$ and $G_{W}$ the image of $\operatorname{Gal}(K)$ in $\operatorname{Aut}_{\mathbf{Q}_{\ell}}(W)$. Then the group $G_{W}$ is not commutative.

Theorem 4.2. Let $X$ be a simple abelian variety over $K$ that is not of CM type. Then for all but finitely many primes $\ell \neq p$ the following condition holds: Let $W$ a nonzero Galois-invariant $\mathbf{F}_{\ell}$-vector space in $X_{\ell}$ and $G_{W}$ the image of $\operatorname{Gal}(K)$ in $\operatorname{Aut}_{\mathbf{F}_{\ell}}(W)$. Then the group $G_{W}$ is not commutative.

Proof of Theorems 4.1] and 4.2. The proof of Theorems 2 and 3 in [24, Sect. 3] works literally provided one replaces the reference to 24 , p. 139, Statements 1 and 2 ] by references to Theorem 1.2 (instead of Statement 1) and to Corollaries 2.3 and 2.7 (instead of Statement 2).

Proof of Theorems 2.19 and 2.20. The proofs of Theorems 7 and 8 in 24, Sect. 4] work literally in our case for Theorems 2.19 and 2.20 respectively. (As in the proof of Theorems 4.1] and 4.2 one should replace the reference to [24, p. 139, Statements 1 and 2] by references to Theorem 1.2 and to Corollaries 2.3 and 2.7])

\section{TORSION AND RAMIFICATION IN SOLVABLE EXTENSIONS}

5.1. Let $K$ be an arbitrary field and $\mathcal{O} \subset K$ a discrete valuation ring whose field of fractions coincides with $K$. We write $\mathfrak{p}$ for the maximal ideal of $\mathcal{O}$ and $p$ for the characteristic of the residue field $\mathcal{O} / \mathfrak{p}$. Let $L / K$ be a finite Galois field extension with Galois group $\operatorname{Gal}(L / K)$ and $\mathcal{O}_{L}$ the integral closure of $\mathcal{O}$ in $L$. The following assertion is well known (see, e.g., [31, Ch. 5, Sect. 7-10], [11, Ch. 1, Sect. 7]).

(i) $\mathcal{O}_{L}$ is a principal ideal domain, the set of its maximal ideals is finite and consists of (say) $g$ maximal ideals $\mathfrak{q}_{1}, \ldots, \mathfrak{q}_{g}$ such that

$$
\mathfrak{p O}=\left(\prod_{i=1}^{g} \mathfrak{q}_{i}\right)^{e(L / K)}
$$

where $e(L / K)$ is the (weak) ramification index at $\mathfrak{p}$. The degree $f(L / K)=$ $\left[\mathcal{O}_{L} / \mathfrak{q}_{i}: \mathcal{O} / \mathfrak{p}\right]$ of the field extension $\left(\mathcal{O}_{L} / \mathfrak{q}_{i}\right) /(\mathcal{O} / \mathfrak{p})$ equals the product $f_{0} p^{s}$ where $f_{0}$ is the degree of the separable closure of $\mathcal{O} / \mathfrak{p}$ in $\mathcal{O}_{L} / \mathfrak{q}_{i}$ and $s$ is a nonnegative integer that vanishes if and only if $\mathcal{O}_{L} / \mathfrak{q}_{i}$ is separable over $\mathcal{O} / p$. The integers $f_{0}$ and $s$ do not depend on $i$. The product

$$
e(L / K) \cdot f_{0} p^{s} g=e(L / K) \cdot f \cdot g=[L: K]=\#(\operatorname{Gal}(L / K))
$$

In particular, $e(L / K) \cdot p^{s}$ divides $[L: K]$. The field extension $L / K$ is tamely ramified at $p$ if and only if $\#\left(I\left(\mathfrak{q}_{i}\right)\right)$ is not divisible by $p$, i.e., $e(L / K)$ is not divisible by $p$ and $\mathcal{O}_{L} / \mathfrak{q}_{i}$ is separable over $\mathcal{O} / p$. Here

$$
I\left(\mathfrak{q}_{i}\right) \subset \operatorname{Gal}(L / K)
$$

is the inertia subgroup attached to $\mathfrak{q}_{i}$. 
(ii) The Galois group $\operatorname{Gal}(L / K)$ acts transitively on the set $\left\{\mathfrak{q}_{i} \mid 1 \leq i \leq\right.$ $g\}$. The corresponding inertia subgroups $I\left(\mathfrak{q}_{i}\right) \subset \operatorname{Gal}(L / K)$ are conjugate subgroups of order $e(L / K) p^{s}$ in $\operatorname{Gal}(L / K)$. (See 31, Ch. 5, Sect. 10, Th. 24 and its proof].)

(iii) Let $L_{0} / K$ be a Galois subextension of $L / K$, i.e., $L_{0} / K$ is a Galois field extension and $L_{0} \subset L$. Then $\mathfrak{q}_{i}^{0}=\mathfrak{q}_{i} \cap \mathcal{O}_{L_{0}}$ is a maximal ideal in $\mathcal{O}_{L_{0}}$ that lies above $\mathfrak{p}$. The image of $I\left(\mathfrak{q}_{i}\right)$ under the surjection $\operatorname{Gal}(L / K) \rightarrow$ $\operatorname{Gal}\left(L_{0} / K\right)$ coincides with the inertia subgroup

$$
I\left(\mathfrak{q}_{i}^{0}\right) \subset \operatorname{Gal}\left(L_{0} / K\right)
$$

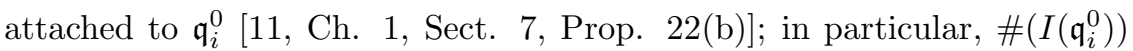
divides \# $\left(I\left(q_{i}\right)\right)$. On the other hand, \# $\left(I\left(\mathfrak{q}_{i}^{0}\right)\right)$ divides $\#\left(\operatorname{Gal}\left(L_{0} / K\right)\right)=$ $\left[L_{0}: K\right]$. This implies that if $\left[L_{0}: K\right]$ and $\#\left(I\left(q_{i}\right)\right)$ are relatively prime then $\#\left(I\left(\mathfrak{q}_{i}^{0}\right)\right)=1$, i.e., $L_{0} / K$ is unramified at $\mathfrak{p}$.

Let $Y$ be an abelian variety of positive dimension over $K$. Let $n \geq 3$ be an integer that is not divisible by $p$. Assume that $Y_{n} \subset Y(K)$, i.e., all points of order $n$ on $X$ are defined over $K$.

Let $\mathcal{Y} \rightarrow \operatorname{Spec}(\mathcal{O})$ be the Néron model of $Y$ [1]; it is a smooth group scheme whose generic fiber coincides with $Y$. Since $Y_{n} \subset Y(K)$, the Raynaud criterion [5], Prop. 4.7] tells us that $Y$ has semistable reduction at $\mathfrak{p}$, i.e., $\mathcal{Y}$ is a semiabelian group scheme.

Let $\ell$ be a prime different from $p$. For all positive integers $j$ the field $K\left(Y_{m_{j}}\right)$ with $m_{j}=\ell^{j}$ is a finite Galois extension. The following assertion was inspired by [8, Ch. XII, Sect. 2.0 on p. 242].

Lemma 5.2. Let $n \geq 3$ be an integer that is not divisible by $p$. Assume that $Y_{n} \subset Y(K)$. Let $L=K\left(Y_{\ell}\right)$ and $\mathfrak{q}$ be a maximal ideal in $\mathcal{O}_{L}$, which lies above $\mathfrak{p}$. Then:

(i) The inertia group $I(\mathfrak{q})$ is a finite commutative $\ell$-group. In particular, $L / K$ is tamely ramified at $\mathfrak{p}$.

(ii) Let $L_{0} / K$ be a Galois subextension of $L / K$. If $\left[L_{0}: K\right]$ is not divisible by $\ell$ then $L_{0} / K$ is unramified at $\mathfrak{p}$.

Proof of Lemma 5.2, The assertion (ii) follows readily from (i). So, let us prove (i).

For all positive integers $j$ let us put $L_{j}=K\left(Y_{m_{j}}\right)$ and $\mathcal{O}_{j}=\mathcal{O}_{L_{j}}$. We have $L_{1}=L, \mathcal{O}_{1}=\mathcal{O}_{L}$. We have a tower of Galois extensions

$$
K \subset L_{1} \subset L_{2} \subset \ldots L_{j} \subset \ldots
$$

We write $L_{\infty}$ for the union $\bigcup_{i=1}^{\infty} L_{i}$. For each $j$ pick a maximal ideal $\mathfrak{q}^{(j)}$ in $\mathcal{O}_{j}$ in such a way that $\mathfrak{q}^{(1)}=\mathfrak{q}$ and $\mathfrak{q}^{(j+1)}$ lies above $\mathfrak{q}^{(j)}$. (Such a choice is possible, because the projective limit of nonempty finite sets is nonempty.) Then the Galois $\operatorname{group} \operatorname{Gal}\left(L_{\infty} / K\right)$ is the projective limit of finite groups $\operatorname{Gal}\left(L_{j} / K\right)$ 's. It is also clear that

$$
\begin{gathered}
\operatorname{Gal}\left(L_{j} / K\right)=\bar{\rho}_{m_{j}, Y}(\operatorname{Gal}(K)) \subset \operatorname{Aut}_{\mathbf{z} / m_{j}} \mathbf{z}\left(Y_{m_{j}}\right), \\
\operatorname{Gal}\left(L_{\infty} / K\right)=\rho_{\ell, Y}(\operatorname{Gal}(K)) \subset \operatorname{Aut}_{\ell}\left(T_{\ell}(Y)\right) \subset \operatorname{Aut}_{\mathbf{Q}_{\ell}}\left(V_{\ell}(Y)\right) .
\end{gathered}
$$

Recall that the natural homomorphisms $I\left(\mathfrak{q}^{(j+1)}\right) \rightarrow I\left(\mathfrak{q}^{(j)}\right)$ are surjective for all $j$. Let $I_{\infty}$ be the projective limit of the corresponding inertia subgroups $I\left(\mathfrak{q}^{(j)}\right)$; clearly, $I_{\infty}$ is a compact subgroup of $\operatorname{Gal}\left(L_{\infty} / K\right)$ and for each $j$ the natural group homomorphism $I_{\infty} \rightarrow I\left(\mathfrak{q}^{(j)}\right)$ is surjective, because the projective limit of nonempty 
finite sets is also nonempty. Therefore one may view $I_{\infty}$ as a certain compact subgroup of

$$
\operatorname{Aut}_{\mathbf{z}_{\ell}}\left(T_{\ell}(Y)\right) \subset \operatorname{Aut}_{\mathbf{Q}_{\ell}}\left(V_{\ell}(Y)\right) .
$$

Since $Y$ has semistable reduction at $\mathfrak{q}$, there exists a $\mathbf{Q}_{\ell}$-vector subspace $\mathbf{W} \subset V_{\ell}(Y)$ such that $I_{\infty}$ acts trivially on $\mathbf{W}$ and $V_{\ell}(Y) / \mathbf{W}$ [5, Prop. 3.5]. It gives us an injective continuous homomorphism of topological groups

$$
\begin{gathered}
\left.I_{\infty} \rightarrow \operatorname{Hom}_{\mathbf{Q}_{\ell}}\left(V_{\ell}(Y) / \mathbf{W}\right), \mathbf{W}\right), \\
\sigma \mapsto\{v+\mathbf{W} \mapsto \sigma(v)-v\} \forall \sigma \in I_{\infty}, v \in V_{\ell}(Y) .
\end{gathered}
$$

Since $I_{\infty}$ is compact, there is a continuous isomorphism between $I_{\infty}$ and its image, which is a compact subgroup of $\operatorname{Hom}_{\mathbf{Q}_{\ell}}\left(V_{\ell}(Y) / \mathbf{W}, \mathbf{W}\right)$. Since the latter is a finite-

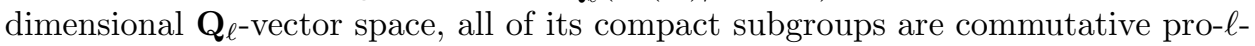
groups (that are isomorphic either to a direct sum of several copies of $\mathbf{Z}_{\ell}$ or to zero). It follows that $I_{\infty}$ is also a commutative pro- $\ell$-group. Since there is a surjective continuous homomorphism

$$
I_{\infty} \rightarrow I\left(\mathfrak{q}^{(j)}\right)
$$

every $I\left(\mathfrak{q}^{(j)}\right)$ is a finite commutative $\ell$-group. This ends the proof, since $\mathfrak{q}=\mathfrak{q}^{(1)}$ and therefore $I(\mathfrak{q})=I\left(\mathfrak{q}^{(1)}\right)$.

Proof of Theorem 2.12. If $K$ is finite then there is nothing to prove. So, let us assume that $K$ is infinite. Let $d \geq 1$ be the transcendence degree of $K$ over $\mathbf{F}_{p}$. Let us pick a positive integer $n \geq 3$ that is not divisible by $p$. Replacing $K$ by $K\left(X_{n}\right)$ and $X$ by $X \times_{K} K\left(X_{n}\right)$, we may assume that $X_{n} \subset X(K)$.

Let $P$ be an infinite set of primes $\ell \neq p$ such that $\tilde{G}_{\ell, X, K}$ is $\ell$-solvable. By deleting finitely many primes from $P$, we may and will assume that the $\operatorname{Gal}(K)$ module $X_{\ell}$ is semisimple for all $\ell \in P$. Since $\tilde{G}_{\ell, X, K}$ is the image of $\operatorname{Gal}(K)$ in $\operatorname{Aut}_{\mathbf{F}_{\ell}}\left(X_{\ell}\right) \cong \mathrm{GL}\left(2 g, \mathbf{F}_{\ell}\right)$, the $\tilde{G}_{\ell, X, K}$-module $X_{\ell}$ is semisimple for all $\ell \in P$.

Let $\mathbf{C}$ be the field of complex numbers. Let us put $g=\operatorname{dim}(X)$. Recall that $X_{\ell}$ is a $2 g$-dimensional $\mathbf{F}_{\ell^{-}}$-vector space. Let $G$ be a finite $\ell$-solvable subgroup of $\operatorname{Aut}_{\mathbf{F}_{\ell}}\left(X_{\ell}\right)$ such that the natural faithful representation of $G$ in $X_{\ell}$ is completely reducible. Let us split the semisimple $\mathbf{F}_{\ell}[G]$-module $X_{\ell}$ into a direct sum

$$
X_{\ell}=\oplus_{i=1}^{m} W_{i}
$$

of simple $\mathbf{F}_{\ell}[G]$-modules $W_{i}$. If $d_{i}=\operatorname{dim}_{\mathbf{F}_{\ell}}\left(W_{i}\right)$ then $2 g==\sum_{i=1}^{m} d_{i}$. By a theorem of Fong-Swan [14, Sect. 16.3, Th. 38], each $W_{i}$ lifts to characteristic zero; in particular, there is a group homomorphism $\rho_{i}: G \rightarrow \mathrm{GL}\left(d_{i}, \mathbf{C}\right)$ whose kernel lies in the kernel of $G \rightarrow \operatorname{Aut}_{\mathbf{F}_{\ell}}\left(W_{i}\right)$ (for all $i$ ). Clearly, the product-homomorphism

$$
\rho=\prod_{i=1}^{d} \rho_{i}: G \rightarrow \prod_{i=1}^{m} \mathrm{GL}\left(d_{i}, \mathbf{C}\right) \subset \mathrm{GL}(2 g, \mathbf{C})
$$

is an injective group homomorphism and therefore $G$ is isomorphic to a finite subgroup of $\mathrm{GL}(2 g, \mathbf{C})$. By a theorem of Jordan [2, Th. Th. 36.13], there is a positive integer $N=N(2 g)$ that depends only on $2 g$ and such that $G$ contains a normal abelian subgroup of index dividing $N$. By deleting from $P$ all prime divisors of $N$, we may and will assume that $\ell$ does not divide $N$ for each $\ell \in P$.

Let us apply this observation to $G=\tilde{G}_{\ell, X, K}$. We obtain that for all $\ell \in P$ the group $\tilde{G}_{\ell, X, K}$ contains an abelian normal subgroup $H_{\ell}$ of index dividing $N$. For 
each $\ell \in P$ let us consider the corresponding subfield of $H_{\ell}$-invariants

$$
K_{\ell}:=\left(K\left(X_{\ell}\right)\right)^{H_{\ell}} \subset K\left(X_{\ell}\right) .
$$

Clearly, $K_{\ell} / K$ is a Galois extension of degree dividing $N$ while $\operatorname{Gal}\left(K\left(X_{\ell}\right) / K_{\ell}\right)$ coincides with commutative (sub)group $H_{\ell}$. Since $K_{\ell} \subset K\left(X_{\ell}\right)$ and $\left[K_{\ell}: K\right]$ divides $N$ and therefore is not divisible by $\ell$, Lemma 5.2 tells us that the Galois extension $K_{\ell} / K$ is unramified with respect to every discrete valuation of $K$. (Since $\operatorname{char}(K)=p$, its every residual characteristic is also $p$.)

Let $k$ be the (finite) algebraic closure of $\mathbf{F}_{p}$ in $K$ and let $S$ be an absolutely irreducible normal $d$-dimensional projective variety over $k$ whose field of rational functions $k(S)$ coincides with $K$. Let $V \subset S$ be a smooth open dense subset such that the codimension of $S \backslash V$ in $S$ is, at least, 2. Let $V_{\ell}$ be the normalization of $V$ in $K_{\ell} / K$. Now Zariski-Nagata purity theorem tells us that the regular map $V_{\ell} \rightarrow V$ is an étale Galois cover; clearly, its degree equals $\left[K_{\ell}: K\right]$ and therefore divides $N$. Let $\pi_{1}(V)$ be the fundamental group of $V$ that classifies étale covers of $V$ (4 , 8, Ch. XII, Sect. 1, pp. 241-242]. This group is a (natural) topologically finitely generated (topological) quotient of $\operatorname{Gal}(K)$ [8, Ch. XII, Sect. 1, p. 242] and the natural surjection

$$
\operatorname{Gal}(K) \rightarrow \operatorname{Gal}\left(K_{\ell} / K\right)
$$

factors through $\pi_{1}(V)$, i.e., it is the composition of the canonical continuous surjection $\operatorname{Gal}(K) \rightarrow \pi_{1}(V)$ and a certain continuous surjective homomorphism

$$
\gamma_{\ell}: \pi_{1}(V) \rightarrow \operatorname{Gal}\left(K_{\ell} / K\right)
$$

whose kernel is an open normal subgroup in $\pi_{1}(V)$ of index dividing $N$.

Since $\pi_{1}(V)$ is topologically finitely generated, it contains only finitely many open normal subgroups of index dividing $N$ (because it admits only finitely many continuous homomorphisms to any finite group of order dividing $N$ ). If $\Gamma$ is the intersection of all such subgroups then it is an open normal subgroup in $\pi_{1}(V)$ that lies in the kernel of every $\gamma_{\ell}$ for all $\ell \in P$; in particular, it has finite index. The preimage $\Delta$ of $\Gamma$ is an open normal subgroup of finite index in $\operatorname{Gal}(K)$ and the corresponding subfield of $\Delta$-invariants $E:=\bar{K}_{s}^{\Delta}$ is a finite Galois extension of $K$ that contains $K_{\ell}$ for all $\ell \in P$. This implies that for all $\ell \in P$ the compositum $E K\left(X_{\ell}\right)$ is abelian over $E$, because $K\left(X_{\ell}\right)$ is abelian over $K_{\ell}$. But $E K\left(X_{\ell}\right)=$ $E\left(X_{\ell}^{E}\right)$ where

$$
X^{E}=X \times{ }_{K} E
$$

is an abelian variety over $E$. Applying Theorem 2.11 to $X^{E}$ and $E$ (instead of $X$ and $K$ ), we conclude that $X^{E}$ is an abelian variety of CM type and isogenous over $\bar{E}=\bar{K}$ to an abelian variety that is defined over a finite field. The same is true for $X$, since $X^{E}=X \times_{K} E$.

\section{Concluding Remarks}

Theorem 1.2 and Corollaries 2.3(iii) and Corollary 2.7 imply readily that the following results remain true for all prime characteristics $p$, including $p=2$.

- Assertions (Sect. 1.3 and 4.4), Corollaries 1-6, Theorem 4.1, and Remark 1 of [20] remain true over any field $E$ that is finitely generated over a finite field of arbitrary characteristic, including 2. Corollary 7 of [20] remains true for any field $F$ of arbitrary prime characteristic, including 2 . 
- Theorem 1.1(ii) of [15].

- Theorems 1.1, 1.4, 1.6, 1.9, 2.1 of [30].

\section{Corrigendum to 29]}

Page 317, Remark 1.3, second sentence: one has to assume additionally that the kernel of the morphism is $W$.

Page 317, line -12: read $\operatorname{Hom}\left(Y^{t}, X^{t}\right)$ instead of $\operatorname{Hom}(Y, X)$.

Page 326, Proof of Theorem 3.4, second line: read $8 g$-dimensional instead of $4 g$-dimensional.

\section{REFERENCES}

[1] Bosch S., Lütkebohmert W., Raynaud M., Néron models, Springer Verlag, Berlin Heidelberg New York, 1990.

[2] Curtis Ch. W., Reiner I., Representation theory of finite groups and associative algebras, Interscience Publishers, New York London, 1962.

[3] G. Faltings, Complements to Mordell, Chapter VI in: Faltings G., Wustholz G. et al., Rational points. Aspects of Mathematics, E6. Friedr. Vieweg \& Sohn, Braunschweig, 1984.

[4] Grothendieck A et al., Revêtements étales et groupe fondamental (SGA 1), Lecture Notes in Math. 224, Springer Verlag, Berlin-Heidelberg-New York, 1971.

[5] Grothendieck A., Modèles de Néron et monodromie, Expose IX dans SGA7 I, Lecture Notes in Math. 288, Springer Verlag, Berlin Heidelberg New York, 1972, 313-523.

[6] Lang S., Algebra, Addison-Wesley Publishing Company, Reading, MA, 1965.

[7] Lenstra H. W.,Jr, Oort F., Zarhin Yu. G., Abelian subvarieties, J. Algebra, 1996, 180, 513516.

[8] Moret-Bailly L., Pinceaux de variétés abéliennes, Astérisque, vol. 129 (1985).

[9] Mumford D., Abelian varieties, Second edition, Oxford University Press, London, 1974.

[10] Oort F., The isogeny class of a CM-type abelian variety is defined over a finite extension of the prime field, J. Pure Applied Algebra, 1973, 3, 399-408.

[11] Serre J.-P., Corps Locaux, Troisieme edition corrigée, Hermann, Paris 1968.

[12] Serre J.-P., Sur les groupes des congruence des variétés abéliennes. Izv. Akad. Nauk SSSR ser. matem., 1964, 28, 3-18; Cuvres II, pp. 230-245, Springer Verlag, Berlin, 1986.

[13] Serre J-P., Abelian $\ell$-adic representations and elliptic curves. Second edition. Addison-Wesley, New York, 1989.

[14] Serre J-P., Représentations linéares des groupes finis, Troisieme edition corrigée, Hermann, Paris, 1978.

[15] Skorobogatov A.N., Zarhin Yu. G., A finiteness theorem for Brauer groups of abelian varieties and K3 surfaces. J. Algebraic Geometry, 2008, 17, 481-502.

[16] Tate J., Endomorphisms of Abelian varieties over finite fields, Invent. Math. 2, 1966, 134-144.

[17] Zarhin Yu. G., Endomorphisms of Abelian varieties over fields of finite characteristic, Izv. Akad. Nauk SSSR ser. matem., 1975, 39, 272-277; Math. USSR Izv. 1975, 9, 255-260.

[18] Zarhin Yu. G., Abelian varieties in characteristic P, Mat. Zametki, 1976, 19, 393-400; Mathematical Notes, 1976, 19, 240-244.

[19] Zarhin Yu. G., Endomorphisms of Abelian varieties and points of finite order in characteristic $P$, Mat. Zametki, 1977, 21, 737-744; Mathematical Notes, 1978, 21, 415-419.

[20] Zarhin Yu. G., Torsion of abelian varieties in fininite characteristic, Mat. Zametki, 1977, 22, 1-11; Mathematical Notes, 1978, 22, 493-498.

[21] Zarhin, Yu. G., Abelian varieties, $\ell$-adic representations and Lie algebras. Rank independence on $\ell$, Invent. Math., 1979, 55, 165-176.

[22] Zarhin, Yu. G., Homomorphisms of Abelian varieties and points of finite order over fields of finite characteristic (in Russian), In: A. L. Onishchik (Ed.), Problems in Group Theory and Homological Algebra Yaroslavl Gos. Univ., Yaroslavl, 1981, 146-147; MR0709632 (84m:14051).

[23] Zarhin Yu. G., A finiteness theorem for unpolarized Abelian varieties over number fields with prescribed places of bad reduction, Invent. Math. 1985, 79, 309-321. 
[24] Zarhin Yu. G., Endomorphisms and torsion of abelian varieties. Duke Math. J., 1987, 54, 131-145.

[25] Zarhin Yu. G., Parshin A. N., Finiteness problems in Diophantine geometry. Amer. Math. Soc. Transl. (2), 1989, 143, 35-102; arXiv 0912.4325 [math.NT] .

[26] Zarhin Yu. G., Hyperelliptic Jacobians without complex multiplication in positive characteristic, Math. Research Letters, 2001, 8, 429-435.

[27] Zarhin Yu. G., Endomorphism rings of certain jacobians in finite characteristic. Matem. Sbornik, 2002, 193:8, 39-48; Sbornik Math., 2002, 193:8, 1139-1149.

[28] Zarhin Yu. G., Non-supersingular hyperelliptic jacobians, Bull. Soc. Math. France, 2004, 132, 617-634.

[29] Yu. G. Zarhin, Homomorphisms of abelian varieties over finite fields, pp. 315-343. In: D. Kaledin, Yu. Tschinkel (Eds.), Higher-dimensional geometry over finite fields, IOS Press, Amsterdam, 2008.

[30] Zarhin Yu. G., Homomorphisms of abelian varieties over geometric fields of finite characteristic, J. Inst. Math. Jussieu, 2013, 12, 225-236.

[31] Zariski O., Samuel P., Commutative Algebra, vol. I, Van Nostrand, Princeton, NJ 1956.

Department of Mathematics, Pennsylvania State University, University Park, PA 16802, USA

E-mail address: zarhin@math.psu.edu 